\title{
Targeting the Hedgehog Signaling Pathway by Glasdegib Limits the Self- Renewal of MDS-Derived Induced Potent Stem Cells (iPSC)
}

\author{
Tetsuzo Tauchi ${ }^{*}$, Seiichi Okabe ${ }^{1}$, Seiichiro Katagiri ${ }^{1}$, Yuko Tanaka ${ }^{1}$, Kaoru Tohyama ${ }^{2}$ and Kazuma Ohyashiki ${ }^{1}$
}

${ }^{1}$ Department of Hematology, Tokyo Medical University, Tokyo, Japan

${ }^{2}$ Kawasaki Med School, Kurashiki, Japan

\begin{abstract}
Objective: Myelodysplastic syndromes (MDS) are clonal hematopoietic disorders characterized by no efficient hematopoiesis and frequent progression to acute myeloid leukemia (AML). Even in low risk MDS, clonal hematopoiesis already dominates at diagnosis, and clones found in secondary AML originate from the MDS stage of disease, highlighting the need to specifically target the MDS-initiating clone. Glasdegib is a potent and selective hedgehog pathway inhibitor that acts by binding Smoothened (SMO) and blocking signal transduction. Glasdegib demonstrated preliminary antitumor activity in a phase I trial, when given as monotherapy in patients with severa hematopoietic malignancies. In the present study, we investigated the molecular mechanisms by which glasdegib regulate the self-renewal of MDS-derived iPS cells (iPSCs) in vivo.
\end{abstract}

Methods: We generated iPSCs from bone marrow mononuclear cells of an MDS patient with chromosome 5 deletion and complex karyotypic abnormalities. We examined the activity of glasdegib against MDS-derived iPSCs transferred NOD/SCID mice in vivo and in vitro. We performed the serial in vivo transplantation to assess the effects of hedgehog inhibition on long term self-renewal. NOD/SCID mice were injected with MDS-iPSCs then treated with glasdegib.

Results: We observed that glasdegib significantly reduced the self-renewal of NOD/SCID re-populating cells from MDS-derived iPSC during serial transplantation in vivo. Further, NOD/SCID mice were injected with MDS-iPSCs, and then treated with glasdegib on day 21 for 7 days. These treatments reduced the population of CD34+CD38-cells. To investigate the entire the apoptosis-induction pathways by hedgehog inhibition, MSD-L cells were incubated with 5-azacytidine and glasdegib for $72 \mathrm{hrs}$. Glasdegib enhanced the expression of cleaved PARP cleaved caspase-3, p21 and reduced expression of c-Myc.

Conclusion: Our pre-clinical results indicate that glasdegib have potential as an important option for controlling the drug-resistant MDS-initiating cells. It is expected that the glasdegib may become extremely useful therapeutic interventions in a number of hematological neoplasms, including MDS, where the persistence of cancer stem cells.

Keywords: Targeted therapy; Hedgehog; MDS; Leukemia Stem Cell; iPSC; NOD/SCID; Phase I

\section{Introduction}

The majority of hematological disorders involving the myeloid lineage are thought to be of stem cell origin, including myeloproliferative disease, myelodysplastic syndrome (MDS), acute myeloid leukemia (AML) and acquired or heritable bone marrow failure syndromes $[1,2]$. In each instance, dysregulation of normal stem cell function is thought to contribute to the disease phenotype [2]. Moreover, stem cell characteristics are modulated by a variety of developmental pathways and regulators. MDS are clonal hematopoietic disorders characterized by in inefficient hematopoiesis and frequent progression to AML [3]. Even in low-risk MDS, clonal hematopoiesis already dominates at diagnosis, and clonal hematopoiesis and clones found in secondary AML originate from the MDS stage of disease [3], highlighting the need to specifically targeted the MDS-initiating clone. Although massive parallel sequencing studies have revealed a number of genomic alterations associated with MDS, functional consequence of these alterations remain poorly understood, mainly due to a difficulty in the ex vivo culture of primary MDS cells and lack of appropriate animal model [4]. The discovery of key transcription factors enabling reprograming a somatic cell into a pluripotent stem cell, called induced pluripotent stem cell (iPSC) open new avenues in medicine [5-7]. Since iPSC can be maintained indefinitely in vitro, they represent an unlimited source of cells, which could overcome the difficulty of obtaining sufficient amounts of MDS cells [8]. Thus, iPSCs become an attractive model for cancer stem cell studying, especially the LSC behavior and the screening of new therapeutic targets reducing LSC survive. Glasdegib is a potent and selective hedgehog pathway inhibitor that acts by binding Smoothened (SMO) and blocking signal transduction (Figureure 1A) [9]. In xenograft models of human colorectal and pancreatic cancer, treatment with glasdegib in combination with other anticancer agents reduced the tumor growth [10]. Furthermore, glasdegib demonstrated preliminary antitumor activity in a phase I trial, when given as monotherapy in patients with several hematopoietic malignancies $[11,12]$. In the present study, we investigated the molecular mechanisms by which glasdegib regulate the self-renewal of MDS-derived iPS cells (iPSCs) in vivo. We observed that glasdegib significantly reduced the self-renewal of NOD/SCID re-populating cells from MDS-derived iPSC during serial transplantation in vivo. In vitro clonogenic growth

*Corresponding author: Tetsuzo Tauchi, Department of Hematology, Tokyo Medical University, 6-7-1 Nishishinjuku, Shinjukuku, Tokyo, 160-0023, Japan, Tel: 81333426111; Fax: 81353816651; E-mail: tauchi@tokyo-med.ac.jp

Received May 16, 2017; Accepted June 13, 2017; Published June 15, 2017

Citation: Tauchi T, Okabe S, Katagiri S, Tanaka Y, Tohyama K, et al. (2017) Targeting the Hedgehog Signaling Pathway by Glasdegib Limits the Self-Renewa of MDS-Derived Induced Potent Stem Cells (iPSC). J Cancer Sci Ther 9: 479-484. doi: 10.4172/1948-5956.1000462

Copyright: (C) 2017 Tauchi T, et al. This is an open-access article distributed under the terms of the Creative Commons Attribution License, which permits unrestricted use, distribution, and reproduction in any medium, provided the original author and source are credited. 
upon serial re-plating, secondary colony formations were significantly inhibited by hedgehog pathway inhibitors. These results suggested that the hedgehog inhibitors may become extremely useful therapeutic interventions in a number of hematological neoplasms, including MDS, where the persistence of cancer stem cells $[13,14]$.

\section{Material and Methods}

\section{Cell line and antibodies and reagents}

MDS-L cell line was established from MDS patient with del (5q) and complex karyotypic abnormalities [15]. Anti-PARP Ab, anticleaved Caspase-3 Ab, anti-p21 Ab, and ant-c-Myc Abs were purchased from Santa Cruz Biotechnology, Inc. (Santa Cruz, CA). Glasdegib was obtained from Pfizer. 5-axacytidine was obtained from Wako (Tokyo).

\section{Generation and culture of iPSCs from a patient with MDS}

This research was approved by the ethics committees at Tokyo Medical University. Primary samples of MDS bone marrow cells were obtained after informed consent. Briefly, mononuclear cells were isolated by Ficoll gradient. CD34+ cells were purified according to the manufacturer's instructions (Milteny Biotech). iPSCs generation were obtained by transduction of CD34+ cells from MDS patients with CytoTune iPS2.0 Sendai virus vector (DINAVEV, Tsukuba, Japan) at a multiplicity of infection (MOI) of 100. After an additional 2-days culture, cells were transferred onto mitomycined mouse embryonic fibroblasts (MEFs) and cultured in ES medium as described below. Starting from day 14 to 21 , the individual iPSC colonies were picked up for expansion.

\section{Human iPSCs culture}

Human iPSC clones were maintained as undifferentiated cells in cultures with mitomycined MEFs. The ES medium used was from ReproStem (ReproCELL Inc., Tokyo) containing 20\% knockout serum replacement (KSR; ReproCELL Inc.), and $5 \mathrm{ng} / \mathrm{ml}$ of recombinant human basic fibroblast growth factor (FGF; ReproCELL Inc.). The ES medium was changed every day.

\section{iPSCs characterization}

For teratoma induction, iPSCs were plated in a $10 \mathrm{~cm}$ MEFs feeder dish. At day 6, approximately $2 \times 10^{6}$ cells were harvested, resuspended in $100 \mu \mathrm{L}$ of ES medium and injected into 6-week-old female NOD/SCID mice subcutaneously. This study was carried out in strict accordance with the recommendation of the Institutional Animal Care and Use Committee in Tokyo Medical University and approved (agreement number: H-27020). Teratomas were harvested 6 to 8 weeks after injection. Paraffin-embedded tissue was sliced and stained with H.E.

\section{Karyotyping}

G-banding analysis was performed following the international system for human cytogenetic nomenclature (ISCN) 2013. Spectral Karyotyping (SKY) was performed with Spectral Karyotyping reagents (SKYPaint $^{\mathrm{TN}}$, Applied Spectral Imaging Ltd., Israel).

\section{RT-PCR analysis}

After extraction of total RNA with RNAeasy reagents (QIAGEN, Germantown, MD), reverse transcription was performed with SuperScript III (Invitrogen, Waltham, MA). Primer sequences used for the detection of stem cell genes were previously describe $[16,17]$.

\section{2nd transplantation}

NOD/SCID mice were injected with 5 colonis of MDS-iPSCs subcutaneously, then treated with glasdegib (100 mg/kg; p.o.; q.d.) $(n=6)$ on day 7 for 21 days. After 56 days, we observed that all mice were alive without body weigh loss. We isolated human CD45+ cells from the spleen of mice and injected equivalent numbers of leukemia cells into secondary recipients. On 28 days, all mice were sacrificed for evaluated.

\section{Results}

\section{Glasdegib enhanced the induction of apoptosis with 5-azacytidine in MDS-L cells}

We used the MDS-L, a myelodysplastic cell line established from an MDS patient with del (5q) and complex karyotypic abnormalities for in vitro studies. MDS-L cells are cultured with indicated concentrations of glasdegib for 72 hrs, extracted RNA were analyzed by Real time PCR. Treatment with glasdegib reduced the expression of Glil and Nanog at more than $5 \mathrm{nM}$ (Figure 1B). Next, MDS-L cells were cultured with 5 -azacytidine and glasdegib for $72 \mathrm{hrs}$, then, the induction of apoptosis was analyzed by Annexin V (Figure 1C). The treatment with 5-azacytidine and glasdegib enhanced the induction of apoptosis in MDS-L cells. To investigate the entire the apoptosis-induction pathways by hedgehog inhibition, MSD-L cells were incubated with 5 -azacytidine and glasdegib for $72 \mathrm{hrs}$, the cell lysates were immunoblotted with antiPARP, anti-cleaved Caspase-3, p21, and c-Myc. Glasdegib enhanced the expression of cleaved PARP, cleaved caspase-3, p21 and reduced expression of c-Myc (Figure 1D).

\section{Establishment of in vivo differentiation system of MDS-iPSCs}

After obtaining informed consent, CD34+ cells were purified from bone marrow mononuclear cells of the MDS (RAEB2) patient, harboring 45,XY,-5, add(7)(q22), +8,-12, add(18)(q22),-12,-20. MDS$\mathrm{CD} 34+$ cells were cultured with $\alpha$-minimum essential medium (MEM) containing $20 \%$ fetal calf serum (FCS) supplemented with $100 \mathrm{ng} /$ $\mathrm{ml}$ stem cell factor, $10 \mathrm{ng} / \mathrm{ml}$ thrombopoietin, $100 \mathrm{ng} / \mathrm{ml} \mathrm{FL3L,} 10$ $\mathrm{ng} / \mathrm{ml} \mathrm{IL-3,} \mathrm{and} 100 \mathrm{mg} / \mathrm{ml} \mathrm{IL-6} \mathrm{for} 2$ days, then, CytoTune iPS2.0 Sendai virus vector transduction with the transcription factors OCT3/4, SOX2, KLF4, and MYC was performed. Two days after transduction, we reseeded cells onto mitomycin treated mouse embrionic fibroblast (MEF) cells and cultured them for another 2 days. Then, we replaced the medium with human ES medium supplemented with $5 \mathrm{ng} / \mathrm{ml}$ of recombinant human bFGF. As a result, MDS-derived iPSCs (MDSiPSCs) were generated, which were derived from the patient (Figure 2A ). MDS-iPSCs showed the typical morphology as iPSCs (Figure 2A) and expresses the embryonic stem cell characteristic transcripts (OCT3/4, NANOG, KLF4, SOX) confirmed by RT-PCR (Figure 2B). SKY fluorescence in situ hybridization (FISH) confirmed the same karyotic anomaly as the patient sample (Figure 2C). Teratoma formation capacity of iPSCs, which were injected into immunodeficient NOD/SCID mice, were also confirmed (Figure 2D). iPSCs were plated in a $10 \mathrm{~cm}$ MEFs feeder dish. At day 6, approximately $2 \times 10^{6}$ cells were harvested, resuspended in $100 \mu \mathrm{L}$ of ES medium and injected into 6-week-old female NOD/SCID mice subcutaneously. All mice demonstrated engraftment of CD34+CD38-cells, CD45+CD19+ cells, and $\mathrm{CD} 45+\mathrm{CD} 3+$ cells from the teratoma at mesoderm portion by flow cytometry (Figure 3A and 3B). NOD/SCID mice were injected with MDS-iPSCs. After 56 days, we isolated human CD45+ cells from the teratoma of mice and injected equivalent numbers of CD45+ cells into secondary recipients. Following 56 days, all mice received MDS-iPSCs derived CD45+ cells engrafted with engraftment of CD34+CD38- 
Citation: Tauchi T, Okabe S, Katagiri S, Tanaka Y, Tohyama K, et al. (2017) Targeting the Hedgehog Signaling Pathway by Glasdegib Limits the SelfRenewal of MDS-Derived Induced Potent Stem Cells (iPSC). J Cancer Sci Ther 9: 479-484. doi: 10.4172/1948-5956.1000462

A

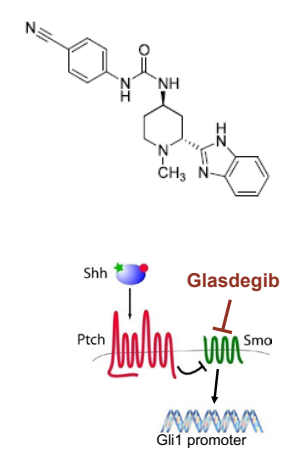

B

PF-04449913

(Glasdegib)
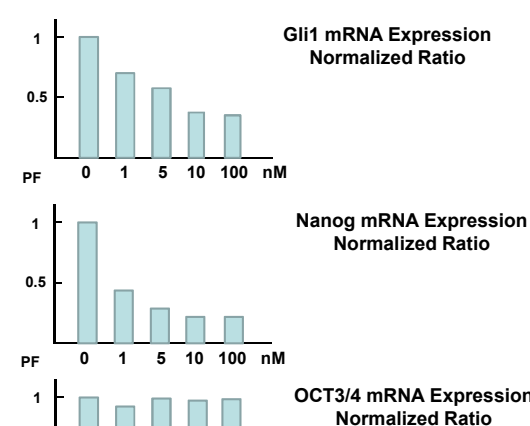

Normalized Ratio

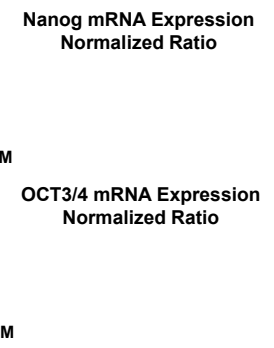

C

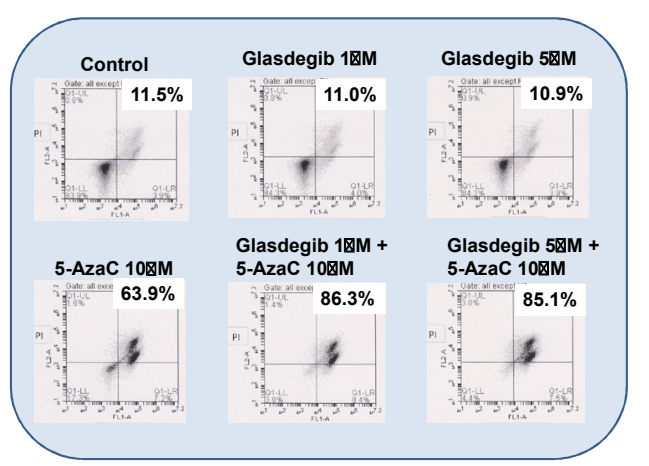

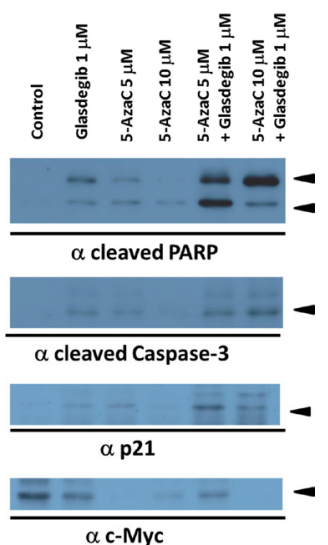

Figure 1: Hedgehog pathway inhibition enhances the induction of apoptosis in MDS-derived cell line. (A) Chemical structure of glasdegib, a selective smoothened antagonist. (B) MDS-L cells are cultured with indicated concentrations of glasdegib for 72 hrs, extracted RNA were analyzed by Real time PCR. (C) MDS-L cells were cultured with 5-azacytidine and glasdegib for $72 \mathrm{hrs}$, then, the induction of apoptosis was analyzed by Annexin V. (D) MSD-L cells were incubated with 5-azacytidine and glasdegib for $72 \mathrm{hrs}$, the cell lysates were immunoblotted with anti-PARP, anti-cleaved Caspase-3, p21, and c-Myc.

cells, CD45+CD19+ cells, and CD45+CD3+cells by flow cytometry (Figure $3 \mathrm{~A}$ and $3 \mathrm{~B}$ ). These results demonstrated that MDS-iPSCs derived $\mathrm{CD} 34+\mathrm{CD} 38$-cells have the self-renewal and mutilineage differentiation capabilities.

\section{Hedgehog inhibition with glasgegib limits self-renewal of MDS-derived iPSC in vivo and in vitro}

We examined the effects of hedgehog pathway modulation on in vitro clonogenic growth. MDS-L cells and the CD34+cells derived from the MDS-iPSCs cells were treated with $1 \mu \mathrm{M}$ of glasdegib for $72 \mathrm{hrs}$, washed free of drugs, and plated in quadruplicate in methylcellulose. At 14 days, colonies were counted as initial plating (Figure 4A ). The representative plate was then washed and cells were resuspended and replated. After an additional 14 days, colonies were counted as secondary replanting (Figure 4A). Upon serial replating, secondary colony formations were significantly inhibited by glasdegib (Figure 4A). We also examined the secondary replating assays by using CD34+cells separated from MDS-RAEB2 primary samples. Clonogenic recovery of MDS-L cells, CD34+ cells derived from MDS-iPSCs, CD34+cells from primary MDS cells were reduced by glasdegib-treatments (Figure 4A). Further, we performed the serial in vivo transplantation to assess the effects of hedgehog inhibition on long term self-renewal (Figure 4B). NOD/SCID mice were injected with MDS-iPSCs then treated with glasdegib on day 7 for 21 days. All mice demonstrated engraftment of CD45+ cells by flow cytometry.
We isolated human CD45+ cells from the spleen of mice from each treatment group and injected equivalent numbers of leukemia cells into secondary recipients. Following 28 days, all mice received MDSiPSCs from vehicle treated mice engrafted with MDS cells (Figure 4B). In contrast, MDS-engraftment was not detected in recipient mice receiving MDS-iPSCs from initial glasdegib-treated donors (Figure 4B). We have also used smoothened inhibotor, vismodegib for the experiments to rule out any off-target effects of glasdegib. We performed the serial in vivo transplantation to assess the effects of another smoothened inhibotor, vismodegib on long term self-renewal (Suplymentary Figure). NOD/SCID mice were injected with MDSiPSCs then treated with vismodegib on day 7 for 21 days. However, 3 mice have been died for vismodegib toxicity (mainly body weigt loss). Remaining mice demonstrated engraftment of CD45+ cells by flow cytometry. We isolated human CD45+ cells from the spleen of mice from each treatment group and injected equivalent numbers of leukemia cells into secondary recipients. Following 28 days, all mice received MDS-iPSCs from vehicle treated mice engrafted with MDS cells (Suplymentary Figure). In contrast, MDS-engraftment was not detected in recipient mice receiving MDS-iPSCs from initial vismogegib-treated donors (Suplymentary Figure). These results demonstrate the persistent effects of hedgehog inhibition on long term self-renewing MDS-iPSCs. Further, NOD/SCID mice were injected with MDS-iPSCs, then treated with glasdegib on day 21 for 7 days. These treatments reduced the population of CD34+CD38-cells (Figure 
Citation: Tauchi T, Okabe S, Katagiri S, Tanaka Y, Tohyama K, et al. (2017) Targeting the Hedgehog Signaling Pathway by Glasdegib Limits the SelfRenewal of MDS-Derived Induced Potent Stem Cells (iPSC). J Cancer Sci Ther 9: 479-484. doi: 10.4172/1948-5956.1000462

A
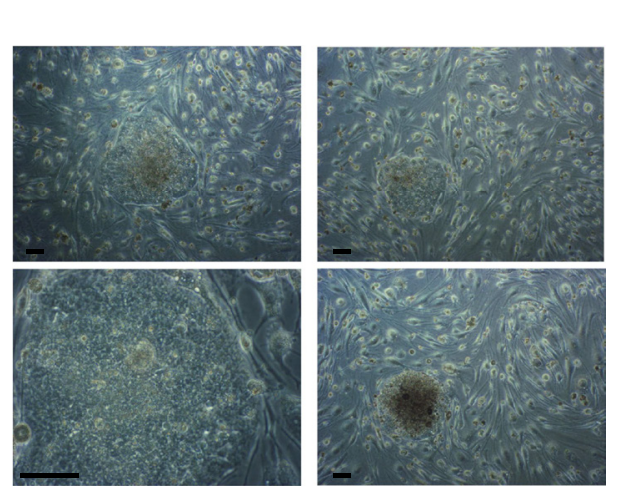

B

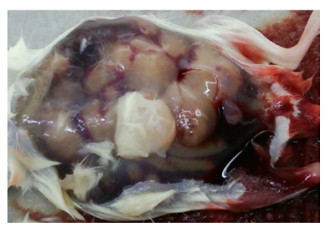

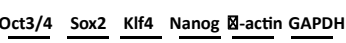

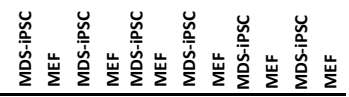

C

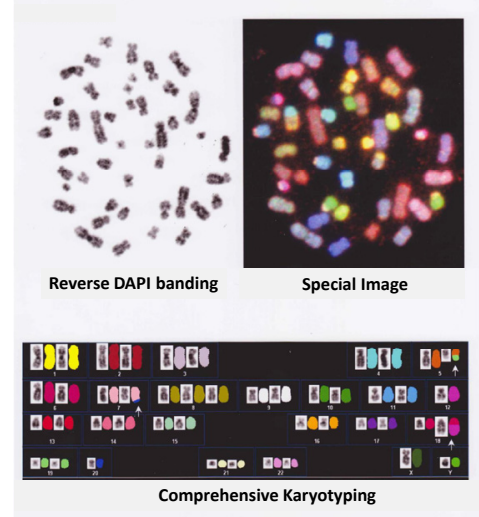

D

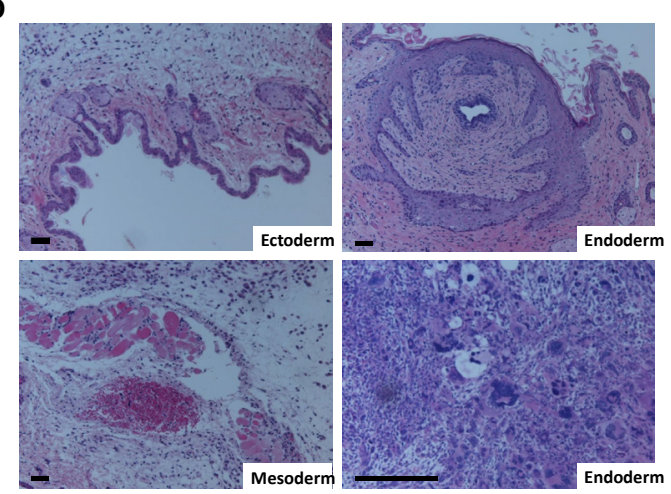

Figure 2: Establishment of iPSCs from MDS patient. (A) iPSCs derived from MDS patients shows the typical morphology as iPSCs. Scale bar=100 $\mu$ m (B) RT-PCR shows the embryonic stem cell characteristic transcripts (OCT3/4, NANOG, KLF4, SOX) in MDS-iPSCs. (C) SKY fluorescence in situ hybridization (FISH) from iPSCs from MDS patient. (D) Histological analyses of teratoma from MDS-iPSCs. Hematoxylin and eosin staining. Original rate of magnification: $x 100$. Scale bar=100 $\mu$ m.

4C). These results demonstrated that the loss of MDS-engraftment in NOD/SCID mice was caused by glasdegib-reduced the population of CD34+CD38-cells.

\section{Discussion and Conclusion}

Efforts to analyze distinct human CSCs have become a major forcus in translational and clinical cancer research. The identification of distinct human CSCs exerting their potential in patients, including in MDS, has remain elusive [2,3]. In vitro modeling of human disease has been greatly facilitated by iPSCs technologies $[8,13]$. Characterized by their ability to self-renew indefinitely and differentiate into various cell lineages, iPSCs provide a powerful system for human disease modeling $[8,13]$. In the present study, we established iPSCs from an MDS patient. MDS-derived-iPSCs had pluripotent markers, teratoma capability, particular morphology with sharp-edged like ESCs, characteristics of iPSC (Figure 2A-2D). In vivo assay using NOD/SCID mice, MDS-derived iPSCs have differentiated into CD34+CD38-cells, CD45+CD19+ cells, and $\mathrm{CD} 45+\mathrm{CD} 3+$ cells from spleen by flow cytometry (Figure $4 \mathrm{~A}$ and 4B). Further, MDS-iPSCs derived CD34+CD38-cells have the self-renewal and mutilineage differentiation capabilities (Figure 3B). However, the reprogramming efficacy of bone marrow MDS CD34+ cells were especially lower than that of normal CD34+ control cells, and delayed reprograming period ( 28 days vs 14 days) (data not shown). This result might be accounted for the fact that cancer-specific genetic lesions may be a hindrance for reprogramming cancer cells illustrated by the rare cases of successful cancer cells reprogramming report. It will be important to overcome the difficulty of reprogram cancer cells. Application of other factors in addition to the Yamanaka factors may be effective, such as exogenous expression of miRNA-302, chemical compound such as histone deacetylate inhibitor, and knock down of p53, p21, and Ink4/Arf [14]. We used Sendai virus system, which is efficient induction of transgene free iPSCs. In retrovirus system, the integration site of retrovirus in iPSCs may affect the gene expression and change the disease phenotype after differentiating them into the original lineage. Much more effort will be required to overcome the difficulty of reprogram cancer cells.

Hedgehog signaling is selectively activated in CSCs compared with bulk tumor cells from several tumor types [10]. Also hedgehog is a highly conserved developmental pathway involved on organogenesis, stem cell maintenance, and tissue regeneration [10]. Glasdegib is a potent and selective hedgehog pathway inhibitor that acts by binding SMO and blocking signal transduction [9]. The phase I study shows that glasdegib is safe and tolerable, with on-target adverse events, doseproportional pharmacokinetics, and early suggestions of clinical activity in patients with hematological malignancies $[11,12]$. 5-azacytidine is a hypomethylating agent that is used as first-line therapy in MDS. However, only $30 \%-50 \%$ of MDS patients show some response. In the present study, we observed that co-treatment with glasdegib and 5 -azacitidine enhanced the induction of apoptosis in MDS-L cells. Serial transplantation is widely accepted as an assay measuring longterm self-renewal in normal hematopoietic stem cells and neoplasms. We used a complementary in vitro assay with serial replating and colony 
Citation: Tauchi T, Okabe S, Katagiri S, Tanaka Y, Tohyama K, et al. (2017) Targeting the Hedgehog Signaling Pathway by Glasdegib Limits the SelfRenewal of MDS-Derived Induced Potent Stem Cells (iPSC). J Cancer Sci Ther 9: 479-484. doi: 10.4172/1948-5956.1000462

A
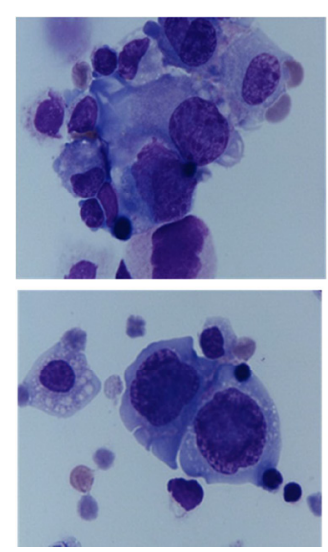
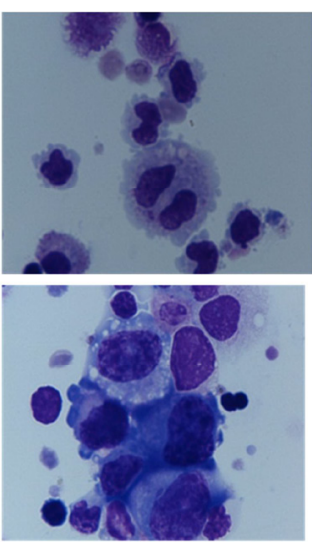

B

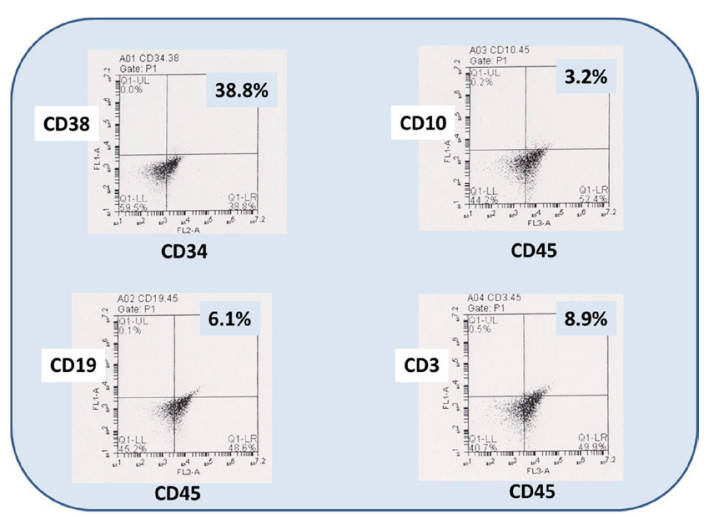

Figure 3: In vivo differentiation system of MDS-iPSCs. (A) Morphology of differentiated MDS-iPSCs. Wright-Giemsa staining. (B) Surface markers of MDS-iPECsderived progenitor cells from MOD/SCID mice. MDS-derived iPSCs have differentiated into CD34+CD38- cells, CD45+CD19+ cells, and CD45+CD3+ cells.

A

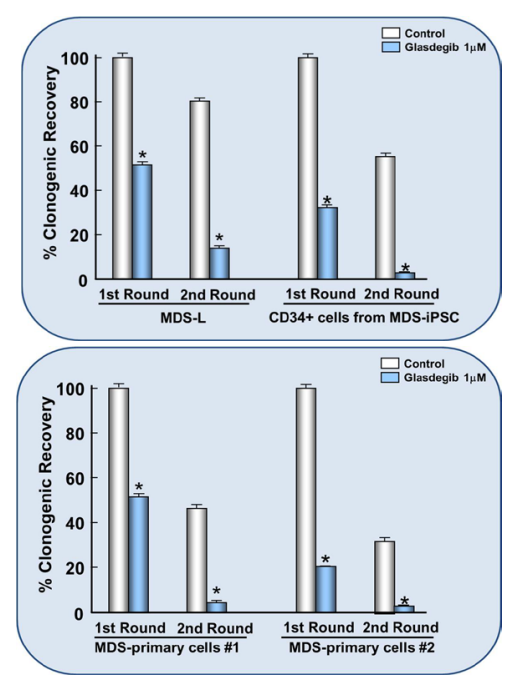

B

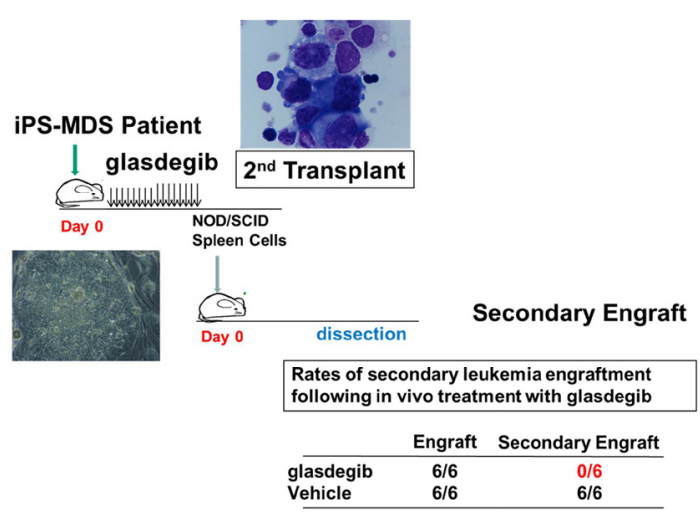

C
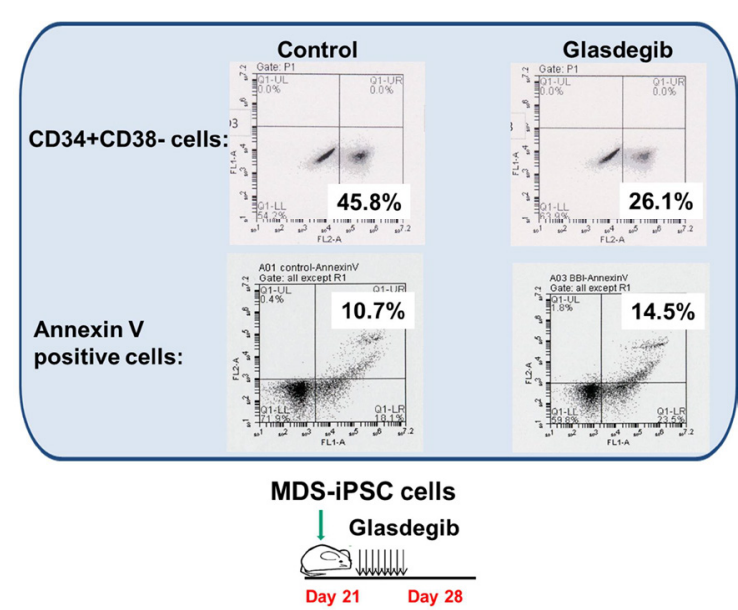

Figure 4: Hedgehog pathway inhibition with glasdegib limits self-renewal in vitro and in vivo (A) MDS-L cells and the CD34+cells derived from the MDS-iPSCs cells were treated with $1 \mu \mathrm{M}$ of glasdegib for $72 \mathrm{hrs}$, washed free of drugs, and plated in quadruplicate in methylcellulose. After an additional 14 days, colonies were counted as secondary replating. Clonogenic recovery of untreated cells was normalized to $100 \%$ and plating results from all treatment groups were expressed as $\%$ control. ${ }^{*} \mathrm{p}<0.001$ compared with control. Similar results were obtained in three independent experiments. (B) An initial cohort of mice was injected with MDS-iPSCs cells and treated with either glasdegib or vehicle. All mice were engrafted with MDS. Following transplantation of harvested spleen cells, leukemia engraftment was not detected in recipient mice receiving MDS-iPSCs cells from initial glasgedib-treated donors. Similar results were obtained in two independent experiments. (C) NOD/SCID mice were injected with MDS-iPSCs cells, then treated with glasdegib on day 21 for 7 days. The percentage of CD34+CD38- cells was analyzed by flow cytometry. 
Citation: Tauchi T, Okabe S, Katagiri S, Tanaka Y, Tohyama K, et al. (2017) Targeting the Hedgehog Signaling Pathway by Glasdegib Limits the SelfRenewal of MDS-Derived Induced Potent Stem Cells (iPSC). J Cancer Sci Ther 9: 479-484. doi: 10.4172/1948-5956.1000462

formation, as well as serial in vivo transplantation by using MDS-iPSCs to assess the effects of hedgehog inhibition on long term self-renewal. We found that glasdegib significantly reduced MDS-iPSCs derived cells and MDS-L cell line as well as primary MDS cells (Figure 3A and 3B). The loss of serial transplantation ability is most consistent with selfrenewal, as similarly seen in serial transplantation experiments with normal hematopoietic cells. It is notable that such long-lasting effects of hedgehog inhibition were seen following only short exposure to glasdegib either 72 hours in colony formation assays or after 28 days of treatment in primary recipient mice. On the basis of our in vitro clonogenic data, we believe that this loss of serial colony formation and transplantation ability is due to the effects of hedgehog inhibition on the self-renewal properties of MDS-initiating cells. However, it is possible that the inhibition of engraftment during secondary transplantation is mediated by the effects of hedgehog inhibition on quiescence of MDSinitiating cells their ability to interact with potential stem cell niches, and proper homing during transplantation. The combined results of cell-based and in vivo studies suggest that glasdegib exhibits sufficient activity against MDS-derived cells to warrant consideration for combination with standard care in MDS. Moreover, clinical importance of glasdegib mediated sensitization of dormant MDS-initiating cells to therapeutic modalities that target dividing cells will need to assess in clinical trials. Although several hedgehog inhibitors have now entered clinical evaluation, it is expected that hedgehog inhibitors may become extremely useful therapeutic innervations in a number of hematopoietic neoplasms where the persistence of cancer stem cells.

\section{References}

1. Jaiswal S, Ebert BL (2014) MDS is a stem cell disorder after all. Cancer Cell 25: 713-714

2. Woll PS, Kjallquist U, Chowdhury O, Doolittle H, Wedge DC, et al. (2014) Myelodysplastic syndromes are propagated by rare and distinct human cancer stem cells in vivo. Cancer Cell 25: 794-808.

3. Pang WW, Pluvinage JV, Price EA, Sridhar K, Arber DA, et al. (2013) Hematopoietic stem cell and progenitor cell mechanisms in myelodysplastic syndromes. Proc Natl Acad Sci USA 110: 3011-3016.

4. Sancho-Martinez I, Nivet E, Xia Y, Hishida T, Agurrie A, et al. (2016)
Establishment of human Ipsc-based models for the study and targeting of glioma initiating cells. Nat comm 7: 1-14.

5. Takahashi K, Yamanaka S (2006) Induction of pluripotent stem cells from mouse embrionic and adult fibroblast cultyres by defined factors. Cell 126: 663-676.

6. Takahashi K, Tanaka K, Ohnuki M, Narita M, Ichisaka T, et al. (2007) Induction of pluripotent stem cells from adult human fibroblasts by defined factors. Cell 131: 861-872.

7. Lee DF, Su J, Kim HS, Chang B, Papatsenko D, et al. (2015) Modeling familia cancer with induced pluripotent stem cells. Cell 161: 240-254.

8. Kotini AG, Chang CJ, Boussaad I, Delrow JJ, Dolezal EK, et al. (2015) Functional analysis of a chromosomal deletion associated with myelodysplastic syndromes using isogenic human induced pluripotent stem cells. Nat biotech 33: 646-655.

9. Munchhof MJ, Li Q, Shavnya A, Borzillo GV, Boyden TL, et al. (2016) Discovery of PF-04449913, a potent and orally bioavailable inhibitor of smoothend. Med Chem lett 3: 106-111.

10. Justilien V, Fields AP (2015) Molecular pathways: Novel approaches for improved therapeutic targeting of hedgehog signaling in cancer stem cells. Clin Cancer Res 21: 505-513.

11. Wagner AJ, Messersmith WA, Shaik MN, Li S, Zheng X, et al. (2015) A phase I study of PF-04449913, an oral hedgehog inhibitor, in patients with advanced solid tumors. Clin Cancer Res 21: 1044-1051.

12. Martinelli G, Oehler VG, Papayannidis C, CourtneyR, Shaik MN, et al. (2015) Treatment with PF-04449913, an oral smoothened antagonist, in patients with myeloid malignancies: A phase 1 safety and pharmacokinetics study. Lance Oncol 8: e339-346

13. Papapetrou EP (2016) Patient-derived induced pluripotent stem cells in cancer research and precision oncology. Nat Med 22: 1392-1400.

14. Lee MR, Prasain N, Chae HD, Kim YJ, Mantel C, et al. (2013) Epigenetic regulation of nanog by miR-302 cluster-MBD2 completes induced pluripotent stem cell reprogramming. Stem Cells 31: 666-681

15. Matsuoka A, Tochiga A, Kishimoto M, Nakahara T, Kondo T, et al. (2010) Lenalidomide induces cell death in an MDS-derived cell line with deletion of chromosome $5 q$ by inhibition of cytokinesis. Leukemia 24: 748-755.

16. Sadarangani A, Pineda G, Lennon KM, Chun HJ, Shih A, et al. (2015) Gli2 inhibition abrogates human leukemia stem cell domancy. J Trans Med 13: 98-112.

17. Liu Z, Zhang J, Kang H, Sun G, Wang B, et al. (2016) Significance of stem cell marker Nanog gene in the diagnosis and prognosis of lung cancer. Oncol Let 12: $2507-2510$. 